\title{
Reconstrucción temprana en el tórax inestable con sistema de osteosíntesis torácico. Reporte de caso
}

\author{
Early unstable chest reconstruction with thoracic osteosynthesis. A case report \\ Enrique Castañeda Saldaña ${ }^{1, a ; 2, b}$, Manolo Elit Briceño Alvarado ${ }^{2, c}$, Cesar Eduardo Del Castillo Peralta 1; \\ 2,d, Fidel Rojas Martinez ${ }^{1 ; 2, \mathrm{~d}}$
}

\section{RESUMEN}

Se presenta el caso de un paciente varón con tórax inestable. Esta lesión es una entidad poco común que puede ser originada por múltiples traumatismos de alto impacto. El diagnóstico se estableció por signos clínicos y estudios de imágenes. El paciente recibió tratamiento quirúrgico con un sistema de osteosíntesis $\operatorname{costal}\left(\operatorname{StraCos}{ }^{\circledR}\right)$; se discuten los resultados obtenidos y sugerencias para casos de este tipo.

PALABRAS CLAVE: Tórax inestable, traumatismos torácicos, fracturas de las costillas. (Fuente: DeCS BIREME).

\section{SUMMARY}

We present the case of a male patient with unstable chest, which is a rare entity that may be caused by high impact trauma. The diagnosis was stablished by clinical manifestations and image results. The patient received surgical treatment with a rib osteosynthesis. We discussed the results obtained and propose suggestions to manage this kind of patients.

KEYWORDS: Flail chest, thoracic injuries, rib fractures. (Source: MeSH NLM).

\section{INTRODUCCIÓN}

El trauma torácico es una patología compleja y de gran importancia debido al incremento de casos vistos en los servicios de emergencia de los hospitales de Lima. El tórax inestable es una causa importante de morbimortalidad en pacientes con lesiones de la caja torácica (1).

Hasta mediados de los 90, el tratamiento no quirúrgico en la inestabilidad torácica incluía maniobras de posicionamiento y tracción de los segmentos fracturados; así como la estabilización interna con presión positiva; sin embargo, todas estas técnicas se complicaban con infecciones debido a la ventilación mecánica prolongada (2).

Tanaka et al (3) en 1988, describieron de forma prospectiva en 37 pacientes los beneficios de la estabilización quirúrgica, entre ellos, una menor morbilidad, menor tiempo de intubación y disminución en costos médicos.

El tratamiento varía según la severidad y complejidad de los componentes, tanto óseos como intraparenquimales, siendo recomendada la

Facultad de Medicina Alberto Hurtado, Universidad Peruana Cayetano Heredia. Lima, Perú.

Departamento de Cirugía, Servicio de Cirugía de Tórax y Cardiovascular. Hospital Cayetano Heredia. Lima, Perú.

Profesor Principal de Cirugía;

Jefe de servicio;

Médico Asistente;

Médico Residente 
fijación costal en casos de tórax inestable o en la fractura de siete arcos costales en un solo hemitórax, independientemente del compromiso esternal, en tanto sea necesaria la reconstrucción de la deformidad de la pared para mantener sus funciones $(4,5)$.

El objetivo de este reporte fue mostrar la estrategia de fijación costal con el sistema StraCos ${ }^{\circledR}$ (Strasbourg Costal Osteosyntheses System, MedXpert GmbH, Heitersheim; Germany), para minimizar errores y optimizar el tratamiento en pacientes con tórax inestable y fracturas múltiples de la región costal.

\section{PRESENTACIÓN DEL CASO}

Varón de 60 años de edad, Natural de Ayacucho, procedente de Lima, quien fue trasladado a emergencia del Hospital Cayetano Heredia, de otra institución por presentar 10 horas antes del ingreso trauma torácico cerrado complicado con hemotórax, y fractura de múltiples costillas en hemitórax izquierdo, producto de la pisada de un toro; asimismo lesión en piel de zona perineal.

$\mathrm{Al}$ examen físico, presión arterial 130/95 $\mathrm{mm} \mathrm{Hg}$, pulso 104 ppm, se encontraba despierto, Glasgow 15, se evidenció palidez muco-cutánea, con oxígeno por máscara de reservorio a 3 litros, presencia de dren torácico izquierdo (drenaje de $1150 \mathrm{ml}$ en $24 \mathrm{~h}$, de

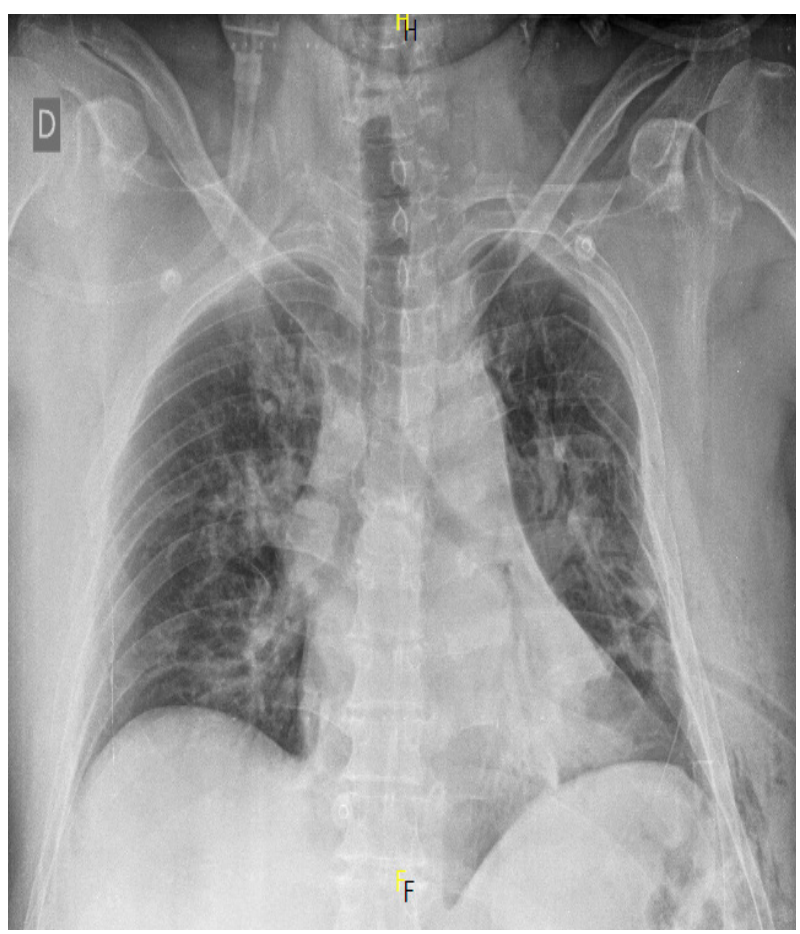

Figura 1. Fractura de múltiples costillas en hemitórax izquierdo.

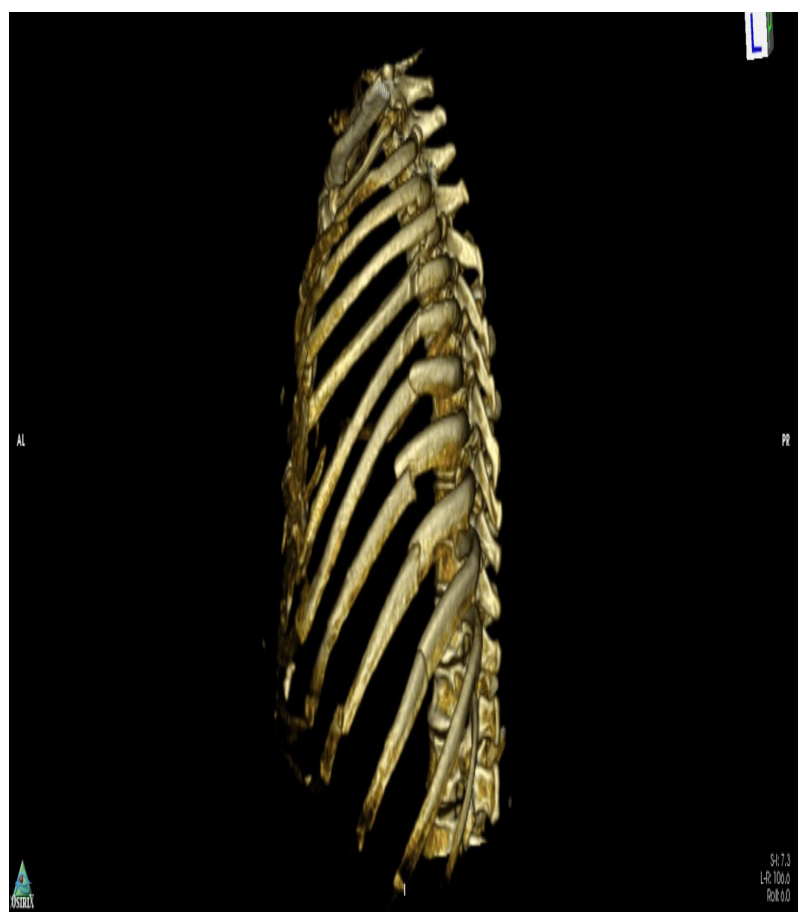

Figura 2. Tomografía axial computarizada con reconstrucción evidenció fractura costal múltiple.

características hemática oscura), aleteo nasal, tiraje supraclavicular, taquipnea y movimiento paradojal del tórax, con murmullo vesicular abolido en $1 / 3$ inferior de hemitórax izquierdo.

La radiografía de tórax mostró imagen compatible con hemotórax y fracturas del tercer al décimo arco costal izquierdo (Figura 1). La tomografía axial computarizada con reconstrucción evidenció contusión pulmonar, hemotórax retenido, fractura costal múltiple, del tercero al décimo arco costal izquierdo (Figura 2).

En la evolución presentó episodio de desaturación e inestabilidad hemodinámica, siendo ingresado al servicio de UCI para la corrección del estado metabólico y el manejo de la vía aérea (ventilación mecánica).

Se determinó su intervención quirúrgica mediante el uso de clips de titanio StraCos ${ }^{\circledR}$. Dentro de los hallazgos se encontraron fracturas múltiples en más de dos segmentos por cada arco costal en hemitórax izquierdo y presencia de hemotórax retenido, por lo cual se procedió a lavado de cavidad y decorticación. Se decidió realizar la fijación de $\operatorname{los} \operatorname{arcos} 7^{\circ}, 8^{\circ}$ y $9^{\circ}$ con clips de titanio, el $8^{\circ}$ requirió dos clips para estabilizar. (Figuras 3 y 4 ). 


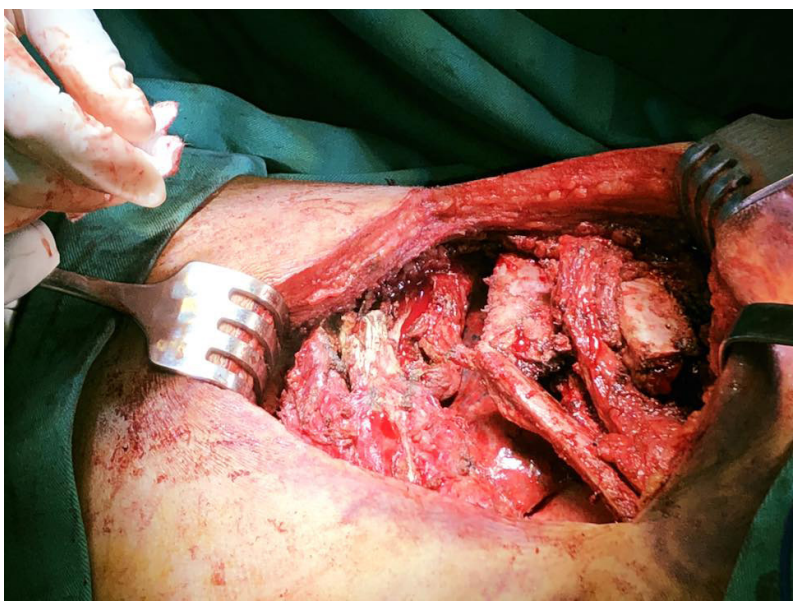

Figura 3. Durante intervención quirúrgica se encontraron fracturas múltiples en más de dos segmentos por cada arco costal en hemitórax izquierdo.

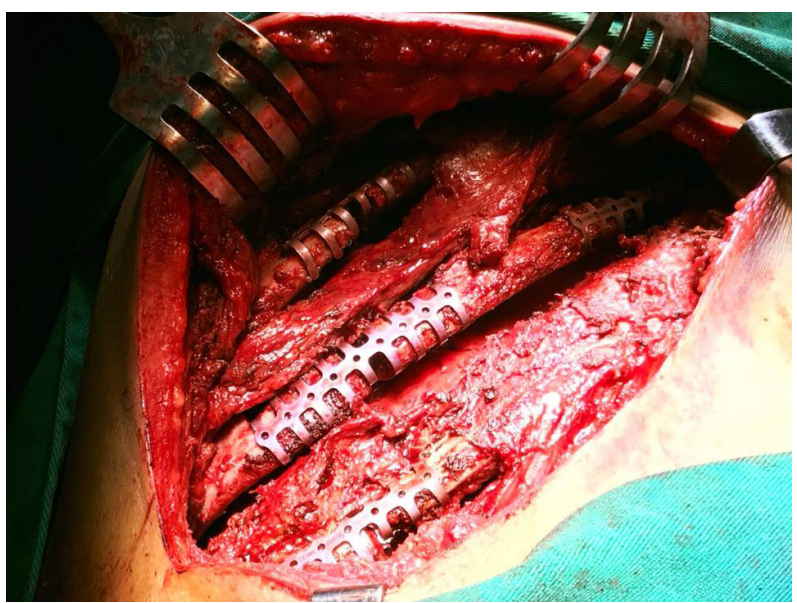

Figura 4. Fijación de los $\operatorname{arcos} 7^{\circ}, 8^{\circ}$ y $9^{\circ}$ con clips de titanio, el $8^{\circ}$ requirió dos clips. Estabilizar.
La evolución postquirúrgica fue satisfactoria, lográndose el destete del ventilador durante las primeras 24 horas, manteniendo una saturación de $\mathrm{O}_{2}$ de $95 \%$ por pulsioximetría con oxígeno ambiental. La evolución clínica fue favorable siendo dado de alta sin mayores complicaciones.

Al control del primer mes la radiografía mostró la amplexación de ambos campos pulmonares y adecuada fijación costal. (Figura 5a, 5b).

\section{DISCUSIÓN}

El tórax inestable, se define como las fracturas consecutivas de tres arcos costales, en dos o más sitios, siendo ésta la forma más compleja de fracturas costales (6). Se estima que el $25 \%$ de las muertes por trauma torácico, están asociadas a lesiones de la caja, entre ellas las fracturas de tres o cuatro costillas en más de dos segmentos de su longitud, las cuales provocan un movimiento paradójico, siendo la localización anterior o lateral los de mayor gravedad por su gran impacto fisiopatológico $(1,6)$. La gravedad se incremente por cada fractura adicional, empeorando el pronóstico en pacientes mayores de 55 años.

Dentro de las complicaciones asociadas se encuentran la contusión pulmonar, con insuficiencia respiratoria, hemotórax, neumonía y el síndrome de dificultad respiratoria aguda $(7,8)$.

El diagnóstico se basa en la evidencia clínica de movimientos paradójicos en la respiración, la evaluación funcional respiratoria mediante el control de gasometría arterial, la crepitación ósea a la
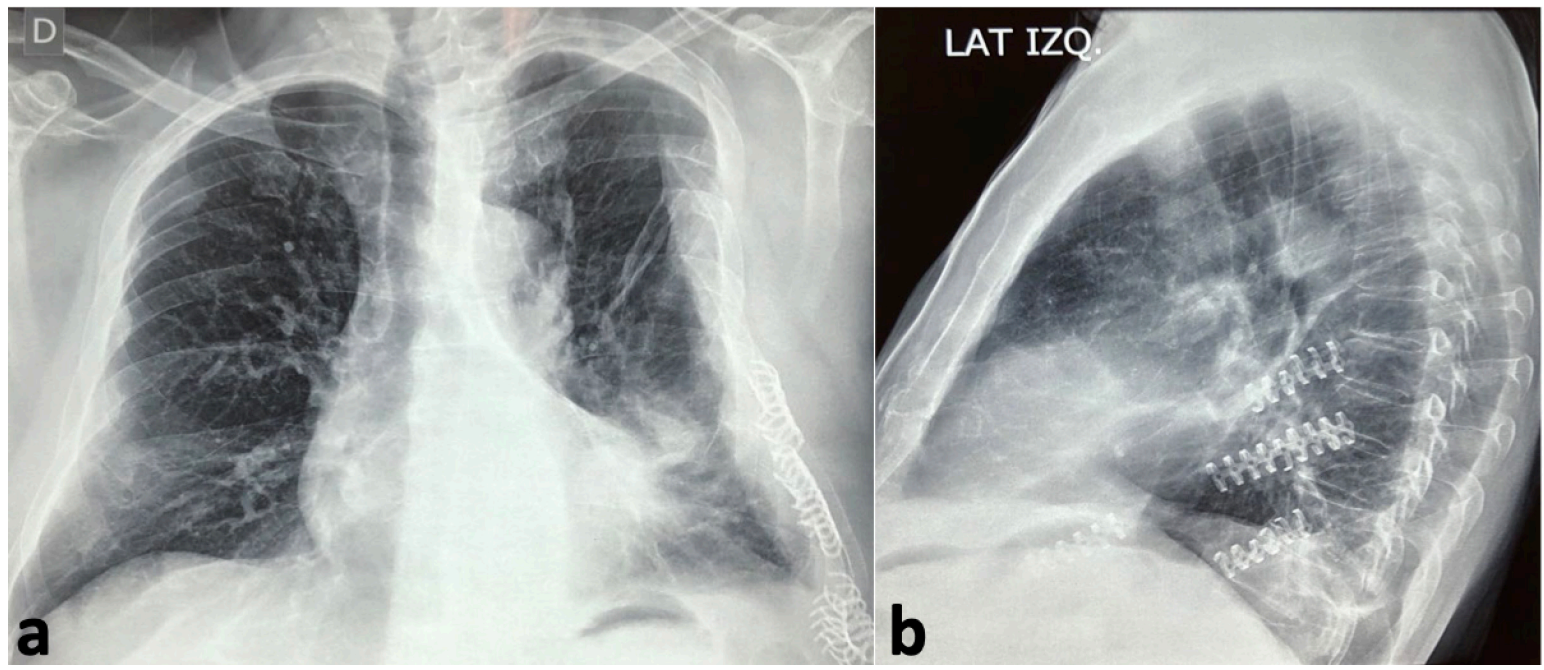

Figura 5. A: Se observa la adecuada amplexación de los campos pulmonares; b: radiografía lateral izquierda que muestra la fijación de los tres arcos costales. 
palpación. Siendo un complemento los estudios de imágenes como las radiografías y tomografías. Este último tiene más sensibilidad para mostrar lesiones como la contusión pulmonar $(9,10)$.

El paciente presentado, fue tratado en la UCI con ventilación mecánica asistida, y colocación de drenaje torácico para el hemotórax. El manejo inicial incluye control del dolor y ventilación mecánica, posteriormente consiste en estabilizar la caja torácica con osteosíntesis (11). El tratamiento tradicional se ha basado en la estabilización interna de ventilación con presión positiva al final de la espiración (12), pero esto condicionaba a tener al paciente en ventilación mecánica por tiempo prolongado mayor a 20 días.

Varios metaanálisis y revisiones sistemáticas, que incluyeron 22 estudios con 988 pacientes con tórax inestable publicados por la Asociación Oriental para la Cirugía de Trauma (EAST), describieron una evolución clínica favorable en aquellos pacientes sometidos a estabilización quirúrgica para el tórax, principalmente en menor tiempo en ventilación mecánica, control del dolor y menos complicaciones infecciosas pulmonares $(11,13-15)$.

Las indicaciones de tratamiento quirúrgico incluyen, la presencia de costillas móviles dolorosas refractarias al manejo del dolor que resultan en una falla respiratoria, deformidad significativa de la caja torácica, falla en el destete de la ventilación mecánica que no sea causada por la contusión pulmonar, inestabilidad y deformidad de la pared torácica o dolor debido a falta de unión o defecto en la unión de fracturas costales (15-17).

Ante la falta de un sistema de puntuación validado o generalizable para predecir el fracaso en las terapias de analgesia, se recomienda la estabilización quirúrgica con el propósito de evitar la intubación en pacientes que están fallando en el tratamiento médico, o ante la necesidad de extubación en pacientes que dependen de la ventilación mecánica debido a inestabilidad o dolor torácico (18).

La cirugía debe realizarse dentro de las primeras 72 horas para poder mitigar el dolor y evitar o disminuir la necesidad del ventilador (14,19-21).

En cuanto al número de costillas que deben ser reparadas, diversos autores recomiendan la fijación quirúrgica de la costilla 4 a 9, debido a que la mayoría del movimiento ocurre entre dichas costillas, con lo cual se produce la mejoría en la función respiratoria general y alivio del dolor $(20,22)$. Un estudio describió que con la fijación del $50 \%$ de las costillas fracturadas, mejorará significativamente la insuficiencia respiratoria y el dolor (20).

Dos estudios de nivel uno demostró que el tratamiento quirúrgico conlleva a una menor estancia en la unidad de cuidados intensivos y disminución de estancia hospitalaria con respecto al manejo conservador (23). Dentro de las diversas técnicas y prótesis de fijación costal, el uso de titanio tiene una mayor biocompatibilidad con los tejidos. A diferencia del acero, es amoldable, libre de tensión sobre el lugar de la fractura y no corrosible, esto lo convierte en una prótesis ideal (24).

Dentro de los sistemas de fijación, el StraCos ${ }^{\circledR}$ es único en el sentido de que no usa tornillos para la fijación, sino que utiliza una serie de clips envolventes para asegurar la placa en las zonas superior e inferior de la costilla. Los resultados en el caso presentado son alentadores, un tratamiento oportuno y con el sistema de osteosíntesis utilizado, predice una evolución favorable en el paciente con tórax inestable; sin embargo, no existen estudios comparativos sobre cuales basar una recomendación para usar un sistema u otro.

\section{Declaración de financiamiento y de conflictos de intereses:}

El reporte fue financiado por los autores; declaran que no existe conflicto de interés alguno.

\section{Contribución de autoría:}

MEBA; CEDP; FRM: Argumentación y la redacción del artículo. ECS: Transferencia tecnológica de técnica quirúrgica a médico asistente y residentes de servicio, revisión critica del artículo.

\section{Correspondencia:}

Dr. Enrique Castañeda Saldaña.

Facultad de Medicina, Universidad Peruana Cayetano Heredia.

Av. Honorio Delgado 430, Lima 31, Perú.

Correo electrónico: enrique.castaneda@upch.pe

\section{REFERENCIAS BIBLIOGRÁFICAS}

1. Sirmali M, Türüt $H$, Topçu $S$, et al. A comprehensive analysis of traumatic rib fractures: morbidity, 
mortality and management. Eur J Cardiothorac Surg $2003 ; 24: 133-8$.

2. Fitzpatrick DC, Denard PJ, Phelan D, Long WB, Madey SM, Bottlang M. Operative stabilization of flail chest injuries: review of literature and fixation options. Eur J Trauma Emerg Surg. 2010; 36: 427433.

3. Tanaka H, Yukioka T, Yamaguti Y, Shimizu S, Goto $\mathrm{H}$, Matsuda H, et al. Surgical stabilization of internal pneumatic stabilization? A prospective randomized study of management of severe flail chest patients. J Trauma. 2002; 52: 727-732.

4. Majercik S, Cannon Q, Granger SR, et al. Longterm patient outcomes after surgical stabilization of rib fractures. Am J Surg 2014; 208:88-92.

5. Bhatnagar A, Mayberry J, Nirula R. Rib fracture fixation for flail chest: what is the benefit? J Am Coll Surg 2012; 215:201-5.

6. Morgenstern $\mathrm{M}$, von Rüden $\mathrm{C}$, Callsen $\mathrm{H}$, et al. The unstable thoracic cage injury: The concomitant sternal fracture indicates a severe thoracic spine fracture. Injury 2016; 47:2465-2472.

7. Mowery NT, Gunter OL, Collier BR, et al. Practice management guidelines for management of hemothorax and occult pneumothorax. J Trauma 2011; 70:510-8.

8. Deunk J, Poels TC, Brink M, et al. The clinical outcome of occult pulmonary contusion on multidetector-row computed tomography in blunt trauma patients. J Trauma 2010; 68(2):387-94.

9. Hauser CJ, Visvikis G, Hinrichs C, et al. Prospective validation of computed tomographic screening of the thoracolumbar spine in trauma. J Trauma 2003; 55:228-34.

10. Plurad D, Green D, Demetriades D, Rhee P. The increasing use of chest computed tomography for trauma: is it being overutilized? J Trauma 2007; 62:631-5.

11. Kasotakis G, Hasenboehler EA, Streib EW, et al. Operative fixation of rib fractures after blunt trauma: A practice management guideline from the Eastern Association for the Surgery of Trauma. J Trauma Acute Care Surg 2017; 82:618-26.

12. Kohn MA, Hammel JM, Bretz SW, Stangby A. Trauma team activation criteria as predictors of patient disposition from the emergency department. Acad Emerg Med 2004; 11(1):1-9.
13. Slobogean GP, MacPherson CA, Sun T, et al. Surgical fixation vs nonoperative management of flail chest: a meta-analysis. J Am Coll Surg 2013; 216(2):302-11.

14. Coughlin TA, Ng JW, Rollins KE, et al. Management of rib fractures in traumatic flail chest: a meta-analysis of randomised controlled trials. Bone Joint J 2016; 98-B:1119-25.

15. Cataneo AJ, Cataneo DC, de Oliveira FH, et al. Surgical versus nonsurgical interventions for flail chest. Cochrane Database Syst Rev 2015; :CD009919.

16. Mayberry JC, Ham LB, Schipper PH, et al. Surveyed opinion of American trauma, orthopedic, and thoracic surgeons on rib and sternal fracture repair. J Trauma 2009; 66:875-9.

17. Dehghan N, de Mestral C, McKee MD, et al. Flail chest injuries: a review of outcomes and treatment practices from the National Trauma Data Bank. J Trauma Acute Care Surg 2014; 76:462-468.

18. Bugaev N, Breeze JL, Alhazmi M, et al. Magnitude of rib fracture displacement predicts opioid requirements. J Trauma Acute Care Surg 2016; 81:699704.

19. Nirula R, Diaz JJ Jr, Trunkey DD, Mayberry JC. Rib fracture repair: indications, technical issues, and future directions. World J Surg 2009; 33:14-22.

20. Pieracci FM, Rodil M, Stovall RT, et al. Surgical stabilization of severe rib fractures. J Trauma Acute Care Surg 2015; 78:883-7.

21. Nirula R, Allen B, Layman R, et al. Rib fracture stabilization in patients sustaining blunt chest injury. Am Surg 2006; 72:307-9.

22. Livingston D, Hauser C. Trauma to the chest wall and lung. In: Trauma, 5th ed, Moore EE, Feliciano D, Mattox K (Eds), McGraw Hill, New York 2004. p.513.

23. Jayle CP, Allain G, Ingrand P, Laksiri L, Bonnin E, HajjChahine J, et al. Flail chest in polytraumatized patients: surgical fixation using Stracos reduces ventilator time and hospital stay. Biomed Res Int. 2015; 2015:624723.

24. Moreno-De La Santa Barajas P, Polo-Otero MD, Delgado Sánchez-Gracián C, Lozano-Gómez $\mathrm{M}$, Toscano-Novella A, Calatayud Moscoso-Del Prado J, et al. Surgical fixation of rib fractures with clips and titanium bars (STRATOS System). Preliminary experience. Cir Esp. 2010; 88:180-186.

Recibido: 16/07/2018

Aceptado: 25/09/2018 